\section{Lung Ultrasound in COPD and Bronchial Asthma-Measurements of Lung Deformation with our Novel Imaging Approach.}

\section{Abstract}

Background: Ultrasound examination pulmonary 3D/4D, elastography and strain rate will constitute a revolution in the coming years. The strain measures the degree of deformation and allows the pulmonary regional and global quantification. It is reproducible and not time consuming. The study aims to evaluate the behavior of the bronchial structure in patients with COPD and asthma using the methods of strain and strain rate (SR).

Methods: The study included critically ill adult patients with early, severe COPD $(n=21)$ and bronchial asthma $(n=12)$ were included retrospectively, as well as healthy controls $(n=11)$. Standard lung ultrasound examinations, including $3 D / 4 D$ análysis, Elastography, were identified and the studies were then reanalysed for assessment of lung strain using speckle-tracking. Lung tracing of the lung was performed in pleural space, pulmonar parenchyma, bronchial estructures and lung vascular estructures determining the longitudinal score and free wall strain in each subject. Spirometry was performed in all patients.

Results: Compared to the control group (healthy subjects) it found that all patients in both groups showed changes the degree of deformation. In $81 \%$ of patients with COPD Spirometry showed a mixed pattern (obstructive and restrictive) and $80 \%$ of them improved by applying bronchial bronchodilator therapy in the time of scanning. $100 \%$ of asthmatic patients had obstructive spirometric pattern and post bronchodilator to treat me there was improvement at all. During the analysis it was found that the values of critical deformation in 12 patients with COPD lines showed increased lung $B$, the expression of pulmonary congestion.

Conclusions: The study shows that longitudinal and global analysis of bronchial deformation can detect measurable changes in COPD and moderate to severe asthma. With increased pulmonary congestion and there is greater presence of lines B values deformation lung is affected. No patients with COPD and Asthma regular strain had worsened.

Abbreviations: 3D/4D: Ultrasound volume scan; B lines: vertical lines pulmonary artifacts; Strain/Strain Rate: deformations scores; SR: strain rate, COPD: chronic obstructive pulmonary disease.
Ernesto Delgado Cidranes', Zuramis Estrada Blanco², Miguel Ángel Fernández Vaquero ${ }^{3}$

1 Founder and Medical Director Advanced Pain Management Center; Ruber Hospital Madrid, Spain

2 Medical Coordinator Advanced Pain Management Center, Sureste Hospital, Madrid, Spain

3 Medical Coordinator Advanced Pain Management Center, Gómez Ulla Military Hospital, Madrid, Spain

Corresponding author:

Ernesto Delgado Cidranes

” edelgadocidranes@gmail.com

Department of Anesthesiology, Pain Medicine and Ultrasound, Ruber Hospital of Madrid, Spain.

Tel: 0034-91-111-9301

Citation: Cidranes ED, Blanco ZE, Vaquero MÁF. Lung Ultrasound in COPD and Bronchial Asthma-Measurements of Lung Deformation with our Novel Imaging Approach. Insights Allergy Asthma Bronchitis. 2015, 1:1.

\section{Introduction}

During recent years, velocity imaging, displacement imaging and deformation imaging (strain and strain-rate imaging) have emerged as valuable tools for more comprehensive and reliable echocardiographic assessment of myocardial function [1,2]. The deformation can also be expressed relative to the length at a previous time instance (natural strain) and in this definition of 
instantaneous strain the reference value is not constant over the time but changes during the deformation process [3].

Lung ultrasound is a basic application of critical ultrasound, defined as a loop associating urgent diagnoses with immediate therapeutic decisions [4].

Ultrasound examination pulmonary 3D/4D (Figure 1) elastography and strain rate will constitute a revolution in the coming years [5]. The strain measures the degree of deformation and allows the pulmonary regional and global quantification. It is reproducible and not time consuming. The study aims to evaluate the behavior of the bronchial structure in patients with COPD and asthma using the methods of strain and strain rate (SR). Spirometry was performed in all patients.

In the right image we can see the air bronchogram 3D/4D. We show bronchial permeability and radial measurement site of a bronchus to integrate it into their analysis of deformation. The measurements are performed in inspiration and expiration. The characteristics of the air bronchogram ultrasound guidance are important in the diagnosis and treatment of lung diseases [6].

When applying the 3D/4D volumes observed in normal lung increased lines A constantly moving up and down following the respiratory movement (bellows type). When the lung has less air and there is a tendency to alveolar collapse laminating lines to decrease and is a sign that could have a future value in the evolution and prediction of lung pathology in apparently normal lung by B-mode sonography $[7,8]$.

The study included critically ill adult patients with early, severe COPD ( $n=21)$ and bronchial asthma $(n=12)$ were included retrospectively, as well as healthy controls $(n=11)$ (Figure 2). Standard lung ultrasound examinations, including 3D/4D análysis, Elastography, were identified and the studies were then reanalysed for assessment of lung strain using speckle-tracking. Lung tracing of the lung was performed in pleural space, pulmonar parenchyma, bronchial estructures and lung vascular estructures determining the longitudinal score and free wall strain in each subject (Figure 3).

\section{Special Remarks}

In the study we have obtained our own reference values for pulmonary strain on the healthy patient. Values that continue being analyzed to achieve a standard that allows us to obtain better results and ensure reproducibility of our research.

The three major strains analyzed

1- Longitudinal overall

2- Overall circumference

3- Radial overall

orrect identification of the pleural space is the key because it is extraordinary element baseline variability in lung tissue. Exploring 3D/4D allows objectively know how deep the bronchial and vascular structures within the lung parenchyma.

This allows knowing the degree of deformation of the structure bronchial and lung parenchyma in inspiration and expiration. The strain used was overall longitudinal and global circumference. The speed measured by tissue Doppler measures the longitudinal velocity (VL), pulmonary components moving towards or away from the transducer (Figure 4).

In all patients with decreasing longitudinal deformation and overall circumference between lung segments it is consistent with the presence of bronchial obstruction in $100 \%$ of cases; provided that the target is in the walls of the bronchial structure.

The lower global longitudinal strain of $-20 \%$ has a negative predictive value $80 \%$ for moderate to severe bronchial obstruction.

\section{Conclusions}

Strain and strain-rate imaging in lung ultrasound is a promising tool for the evaluation of myocardial function.

The study shows that longitudinal and global analysis of bronchial deformation can detect measurable changes in COPD and moderate to severe asthma.

With increased pulmonary congestion and there is greater presence of lines B values deformation lung is affected.

Strain and strain rate data also provide valuable prognostic information, especially in predicting regression to the normal lung function. 


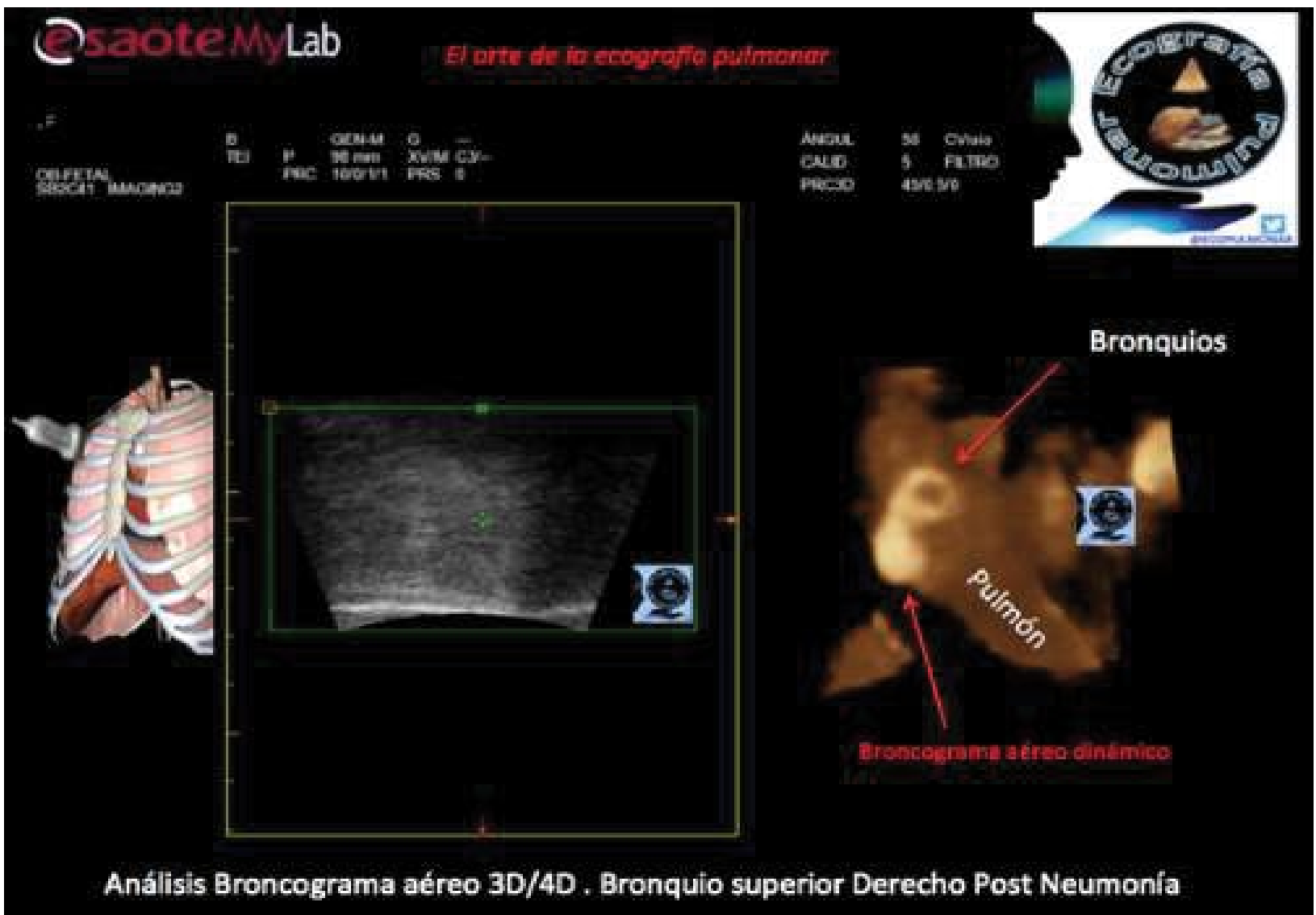

Figure 1 Lung ultrasound volumetric 3D / 4D allows visualization of the lung parenchyma objective; essential to identify the target which we will measure the strain element.

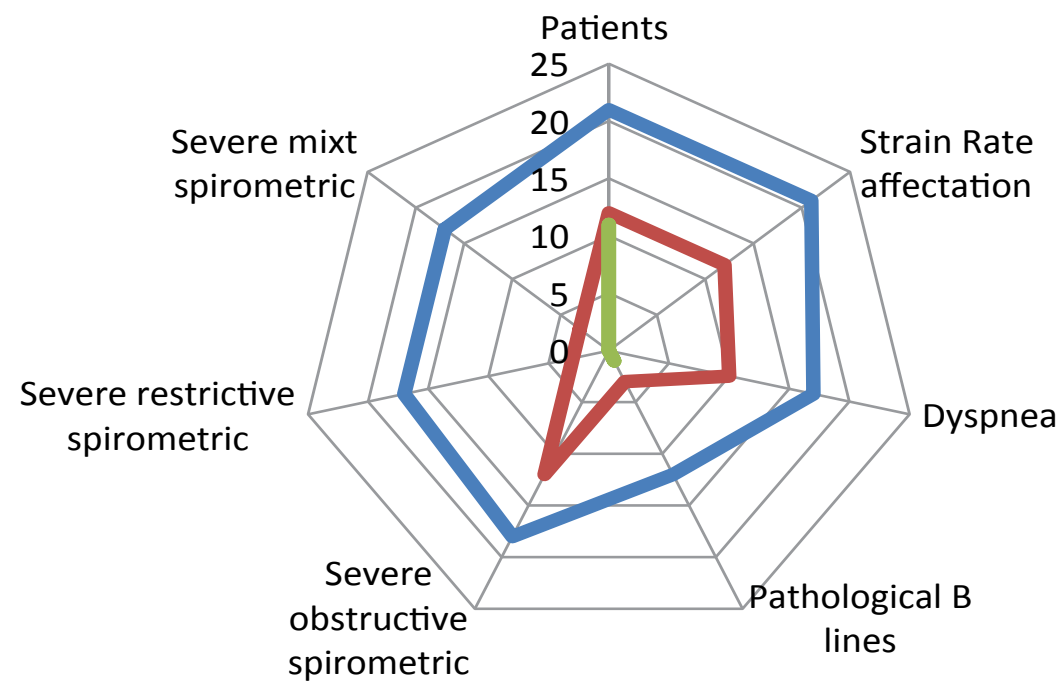

Group 1 COPD

Group 2 Asthma

Group 3 Control

Figure 2 Map of main variables studied. 


\section{Measurements of lung deformation (strain and strain rate)} The art of lung ultrasound

Madrid; Spain

ecopulmonar@gmail.com
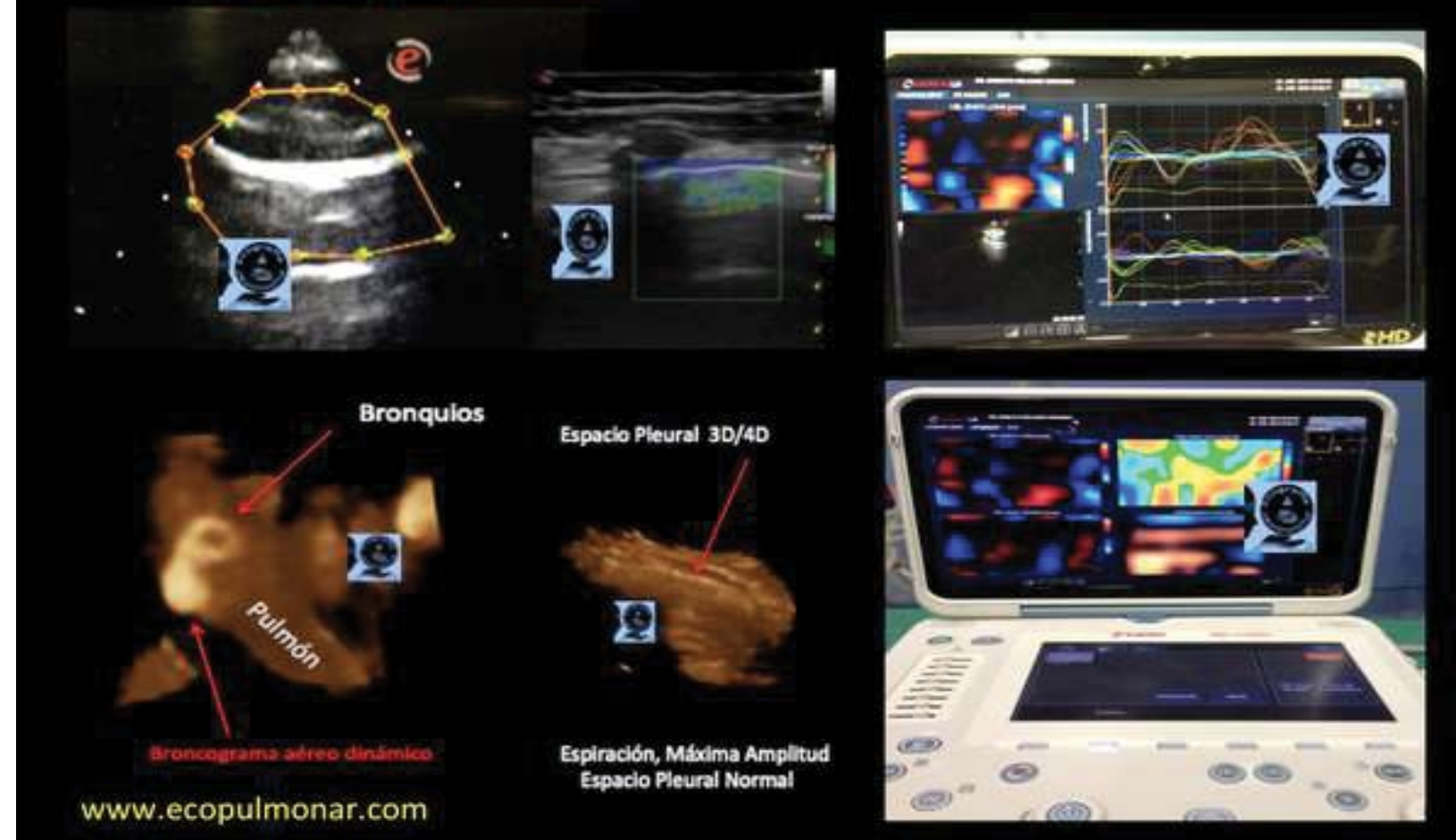

www.ecopulmonar.com

Figure 3 Photographic sequence, strain rate of lung parenchyma, elastography and pulmonary analysis 3D / 4D allowing objectively locate the target of the study. The images on the right show the ultrasound deformation curves during inspiration and expiration.
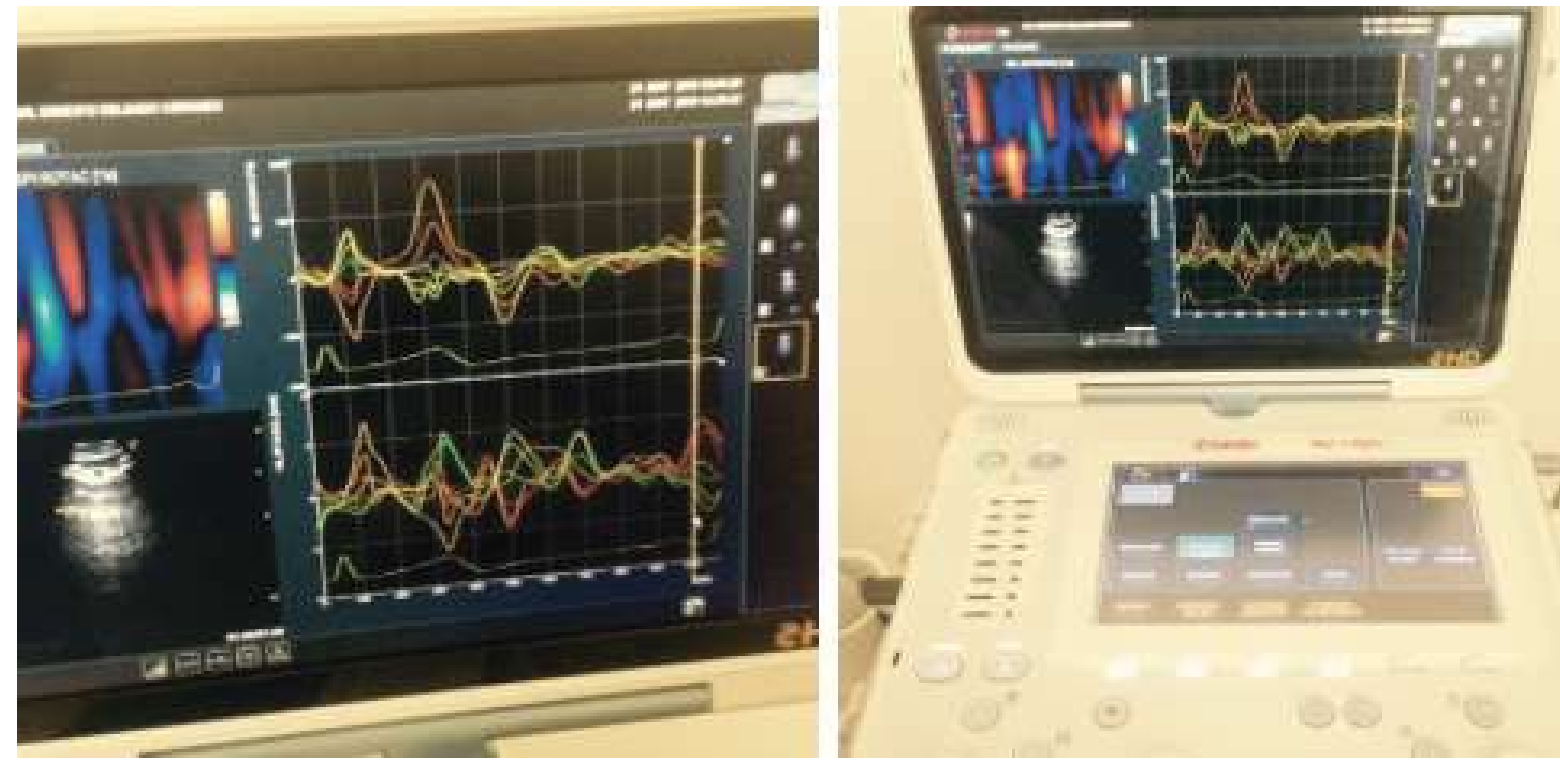

Figure 4 Strain inspiratory and expiratory. 


\section{References}

1 Sheehan $\mathrm{FH}$ (2002) Quantitative evaluation of regional left ventricular systolic function. The Practice of Clinical Echocardiography, WB Saunders Company, Philadelphia pp: 65-87.

2 Yu CM, Sanderson JE, Marwick TH, Oh JK (2007) Tissue Doppler imaging a new prognosticator for cardiovascular diseases. J Am Coll Cardio 20: 234-243.

3 D'Hooge J, Heimdal A, Jamal F (2000) Regional strain and strain rate measurements by cardiac ultrasound: principles, implementation and limitations. Eur J Echocardiogr1: 154-70.

4 Lichtenstein (2014) Lung ultrasound in the critically ill. Annals of Intensive Care 4: 1 .
5 Cidranes ED (2014) Elastography.:Higher Quality and Confidence for Better Characterization of Musculoskeletal Injuries. J Anesth Crit Care Open Access 1: 00003.

6 Lichtenstein D, Mezière G, Seitz (2009) The dynamic air bronchogram. An ultrasound sign of alveolar consolidation ruling out atelectasis. Chest 135: 1421-1425.

7 Volpicelli G, Mussa A, Garofalo G (2006) Bedside lung ultrasund in the assessment of alveolar-interstitial síndrome. Am J Emerg Med 24: 689-696.

8 Slasky BS, Auerbach D, Skolnick ML (1983) Value of portable realtime ultrasound in the intensive care unit. Crit Care Med 11:160-164 